\title{
Nível Socioeconômico e Autopercepção como Parceiro Romântico no Ambiente Universitário
}

\author{
Anthonieta Looman Mafra*,1 \\ Orcid.org/0000-0002-7612-8778 \\ Felipe Nalon Castro ${ }^{2}$ \\ Orcid.org/0000-0002-6690-5182 \\ Fívia de Araújo Lopes ${ }^{2}$ \\ Orcid.org/0000-0002-8388-9786
}

${ }^{I}$ Universidade Potiguar - Laureate International Universities, Natal, RN, Brasil

${ }^{2}$ Universidade Federal do Rio Grande do Norte, Natal, RN, Brasil

\section{Resumo}

De acordo com a perspectiva da Psicologia Evolucionista, existem diferentes padrões de preferência sexual para homens e mulheres. Sabe-se que essas preferências são moduladas pela autopercepção dos indivíduos e que o contexto, por sua vez, pode afetar a mesma. O presente trabalho buscou verificar se o contexto econômico, indicado pelo poder aquisitivo, afeta a autopercepção como parceiro romântico. Para isso, 316 universitários responderam um questionário indicando sua classificação socioeconômica e autopercepção como parceiro romântico. Os resultados indicaram que a autopercepção da condição financeira diferiu de acordo com o nível socioeconômico. Contudo, diferença com relação à percepção da atratividade geral foi maior em homens de classe A comparado a mulheres da mesma classe, enquanto que para a característica determinado/trabalhador houve diferença entre sexos, na qual mulheres avaliaram-se melhor que homens. Os resultados dão suporte à Teoria do Pluralismo Estratégico ao indicar que o ambiente pode influenciar o processo de seleção de parceiros por aumentar a atratividade geral percebida de homens de maior nível socioeconômico em relação aos outros grupos da amostra. Além disso, os resultados sugerem a desigualdade entre os sexos na autopercepção como parceiro romântico.

Palavras-chaves: Autopercepção, Psicologia Evolucionista, estudantes universitários, nível socioeconômico, reprodução humana.

\section{Socioeconomic Level and Self-Perception as a Romantic Partner in a University Setting}

\begin{abstract}
From an Evolutionary Psychology perspective, men and women exhibit different sexual preferences. These preferences are modulated by the self-perception of individuals, which may also be affected by

* Endereço para correspondência: Universidade Potiguar, Pro-Reitoria Academica, Mestrado Profissional em Psicologia Organizacional e do Trabalho, Capim Macio, Natal, RN, Brasil 59082-902. Fone: (84) 32151234. E-mail: looman.anthonieta@gmail.com, castrofn@gmail.com e fivialopes@gmail.com Apoio: Conselho Nacional de Desenvolvimento Científico e Tecnológico (CNPq).
\end{abstract}


context. The present study sought to determine whether economic context, as indicated by purchasing power, affects self-perception as a romantic partner. To that end, 316 university students completed a questionnaire containing questions on socioeconomic status and self-perception as a romantic partner. The results indicated that self-perceived financial situation differed according to socioeconomic level. However, the difference in perceived general attractiveness was higher in class A men than in women from the same economic class, while for the determined/hardworking trait, there was a difference between sexes, in which women perceived that they were better than men. The results support the theory of strategic pluralism, showing that the environment can influence the partner selection process by increasing the general attractiveness perceived by men in the highest economic class (A) compared to the other groups in the sample. Moreover, the results suggest inequality between the sexes in terms of self-perception as a romantic partner.

Keywords: Self-perception, Evolutionary Psychology, university students, socioeconomic level, human reproduction.

\section{Nivel Económico y Auto-Percepción como una Pareja Romántica en el Ámbito Universitario}

\section{Resumen}

De acuerdo con la perspectiva de la Psicología Evolutiva, hay diferentes estándares de preferencias sexuales para hombres y mujeres. Se sabe que estas preferencias son moduladas por la autopercepción de la persona y el contexto, a su vez, puede afectar a la autopercepción. Este estudio buscó determinar si el contexto económico, indicado por el poder adquisitivo afecta a la autopercepción como pareja romántica. Para tal, 316 estudiantes respondieron a un cuestionario indicando su clasificación socioeconómica y la autopercepción como pareja romántica. Los resultados indicaron que la autopercepción de la condición financiera difiere según el nivel socioeconómico. Sin embargo, las diferencias en cuanto a la percepción del atractivo general fue mayor en hombres de classe A en comparación con las mujeres de la misma clase, mientras que para la característica determinado/trabajador hubo diferencias entre los sexos, en la que mujeres calificaron a sí mismas mejor que los hombres. Los resultados dan soporte a la Teoría del Pluralismo Estratégico al indicar que el ambiente puede influenciar el proceso de selección de parejas por aumentar el atractivo general percibido de hombres de mayor nivel socioeconómico en relación a los otros grupos de la muestra. Además, los resultados sugieren la desigualdad entre los sexos en la autopercepción como pareja romántica.

Palabras clave: Autopercepción, brasileños, Psicología Evolutiva, estudiantes universitarios, nivel socioeconómico, reproducción humana.

A Teoria das Estratégias Sexuais prediz que machos e fêmeas tendem a escolher seus parceiros de forma a maximizar o sucesso reprodutivo (Buss \& Schmitt, 1993). Com humanos, não é diferente. Ainda de acordo com os mesmos autores, os indivíduos que possuíam preferências por parceiros com características que indicassem genes favoráveis à transmissão de características desejáveis provavelmente tiveram maior sucesso reprodutivo que aqueles que não as possuíam. De acordo com a perspectiva evolucionista, esse é um dos motivos que possibilitou o surgimento dos padrões comportamentais masculinos e femininos para a busca de parceiros românticos (Gaulin \& McBurney, 2001).

Como a atratividade física fornece importantes pistas acerca da condição física e saúde reprodutiva da mulher (que podem refletir o elevado investimento fisiológico na prole), em geral, homens demonstram maior interesse por mulheres consideradas mais atraentes fisicamente (Buss, 1989; Castro, Hattori, Yamamoto, 
\& Lopes, 2013; Geary, Vigil, \& Byrd-Craven, 2004; Gutierres, Kenrick, \& Partch, 1999; Kenrick, Sadalla, Groth, \& Trost, 1990; Kruger, Fitzgerald, \& Peterson, 2010; Pawlowski, 2000). No entanto, mulheres não são as únicas a investir nos filhos. Apesar do baixo investimento fisiológico, os homens podem fornecer cuidado, proteção e recursos para a parceira e seus filhos (Buss, 1989; Castro \& Lopes, 2011; Geary et al., 2004; Hattori, Castro, \& Lopes, 2013; Pawlowski, 2000). Nesse sentido, características ligadas ao status social tendem a se sobressair nas preferências femininas quando comparadas às preferências masculinas (Buss, 1989; Castro \& Lopes, 2011; Castro et al., 2013; Gaulin \& McBurney, 2001; Geary et al., 2004; Hattori et al., 2013; Pawlowski, 2000). Porém, existem ainda características que possuem importância similar para mulheres e homens quando buscam por um parceiro (Castro \& Lopes, 2011; Hattori et al., 2013; Mafra \& Lopes, 2014). Um exemplo de uma dessas características é fidelidade, a qual é altamente valorizada por homens e mulheres, por garantir àqueles a paternidade (Dosmukhambetova \& Manstead, 2011; Trivers, 1972) e a estas o investimento nelas e em suas proles (Gaulin \& McBurney, 2001).

Além da busca pela satisfação das preferências, para que o relacionamento seja duradouro, pesquisadores defendem a ideia de que se deve encontrar um parceiro com o valor de mercado (valor reprodutivo do indivíduo em um determinado ambiente) similar (Buston \& Emlen, 2003; Castro, Hattori, \& Lopes, 2012; Lee, Loewenstein, Ariely, Hong, \& Young, 2008). Para isso, são necessárias tanto a avaliação do valor reprodutivo dos potenciais parceiros quanto a autopercepção. A autopercepção do valor dos atributos do indivíduo em um dado contexto é afetada pela comparação com o valor percebido dos competidores (Castro et al., 2012; Fisher, Cox, Bennett, \& Gavric, 2008; Pawlowski, 2000).

Buston e Emlen (2003), em uma pesquisa feita com estudantes universitários americanos, confirmaram que indivíduos que se avaliam melhor possuem maiores exigências que aqueles que não se avaliam tão bem ao procurar por um parceiro romântico, o que seria um indicativo de que os pares podem ser formados por pessoas que possuem valor de mercado semelhante (avaliado de forma holística). No mesmo sentido, Lee e colaboradores (2008) encontraram que homens são mais influenciados que as mulheres pela atratividade física de suas parceiras e são menos afetados por quão atraente eles são, dando um indício de que homens dão mais importância a outras características ao se avaliarem como parceiro romântico. Assim, as características mais preferidas em um parceiro romântico de um sexo são as que influenciam mais na autopercepção como parceiro romântico do outro (Lee et al., 2008). Mulheres tendem a realizar sua autopercepção com base na atratividade física das outras mulheres disponíveis no ambiente (Buss \& Shackelford, 2008), enquanto homens tendem a basear sua autopercepção mais em características indicadoras de alto status social (Fisher et al., 2008; Goodwin, Marshall, Fülöp, Adonu, \& Spiewak, 2012).

Além de considerarmos que a autopercepção é baseada no valor de mercado dos demais indivíduos presentes no ambiente (Castro et al., 2012; Fisher et al., 2008), na Teoria do Pluralismo Estratégico, Gangestad e Simpson (2000) propuseram que as estratégias adotadas por homens e mulheres variam de acordo com fatores ambientais, como incidência de patógenos e disponibilidade de recursos. Nesse sentido, o nível socioeconômico de um indivíduo, indicado por seu poder aquisitivo, seria um fator ambiental que pode ocasionar mudança da autopercepção. Esse efeito pode ser mais intenso em países que são marcados pela acentuada diferença econômica com a qual as pessoas de diferentes níveis socioeconômicos se deparam frequentemente. O Brasil possui uma elevada desigualdade de distribuição de renda (coeficiente de Gini elevado, superior a 49,7; Central Intelligence Agency [CIA], 2014), e é um país cuja desigualdade social é elevada, sendo este importante para demonstrar o status social e o respeito que deve se ter em relação a pessoa de maior status social (The Hofstede Centre, 2013). O status social, portanto, indica tanto a possibilidade de possuir patrimônio que um indivíduo pode ter, como a qualidade de vida dele e da sua família (Heo, 
Moser, Chung, \& Lennie, 2012). Assim, o presente estudo buscou investigar o efeito do contexto econômico na autopercepção de homens e mulheres como parceiro para relacionamento romântico dentro de um mesmo ambiente, no caso, o ambiente universitário. No estudo, o contexto econômico foi avaliado por meio da classificação dos participantes em função de seu poder econômico e o ambiente universitário foi escolhido devido à nivelação educacional dos indivíduos, podendo proporcionar uma perspectiva similar de futuro entre os participantes.

\section{Método}

\section{Local do Estudo e Participantes}

A pesquisa foi realizada em cinco instituições de ensino superior da cidade do Natal, Rio Grande do Norte, com um total de 316 estudantes universitários de 18 a 29 anos, sendo destes 109 homens $\left(M_{\text {idade }}=22,05 ; D P_{\text {idade }}=2,74\right.$ anos $)$ e 207 mulheres $\left(M_{\text {idade }}=21,08 ; D P_{\text {idade }}=2,54\right.$ anos), todos heterossexuais ${ }^{1}$. Quase metade da amostra era composta de brancos $(45,6 \%)$, além de $36,7 \%$ de pessoas pardas, $8,5 \%$ de pessoas amarelas, $5,7 \%$ de pessoas com cor de pele preta e $2,5 \%$ de indígenas $(0,9 \%$ dos participantes não responderam à pergunta). Tais percentuais estão de acordo com a classificação populacional apresentada pelo Instituto Brasileiro de Geografia e Estatística (IBGE, 2010). A distribuição quanto ao nível socioeconômico foi a seguinte: $17,72 \%$ corresponde à classe A (renda mensal familiar maior ou igual 13 salários mínimos), 53,80\% corresponde à classe $\mathrm{B}$ (renda mensal familiar de aproximadamente 6 salários mínimos) e 28,48\% à classe $\mathrm{C}$ (renda mensal familiar de dois salários mínimos). Detalhando o nível socioeconômico por sexo, a amostra foi composta por 37 mulheres e 19 homens da classe A, 107 mulheres e 63 homens da classe B e 63 mulheres e 27 homens da classe $\mathrm{C}$.

Foram utilizados apenas participantes heterossexuais devido ao baixo número de participantes não-heterossexuais para que compusessem um grupo que permitisse uma análise em separado.
As classes D (renda mensal familiar de aproximadamente um salário mínimo) e E (renda mensal familiar menor que um salário mínimo) foram retiradas da amostra devido ao baixo número de participantes que se encaixavam nestes níveis socioeconômicos.

\section{Instrumentos}

O questionário utilizado para a obtenção de dados para a pesquisa continha questões de caráter socioeconômico (conforme classificação da Associação Brasileira de Empresas de Pesquisa [ABEP], 2010) e uma parte direcionada à autopercepção do participante enquanto parceiro romântico.

A classificação socioeconômica da ABEP é obtida a partir de um questionário que tem como objetivo saber a quantidade de bens ou serviços que o participante tem em casa. Para isso, os itens do questionário são: televisores coloridos, aparelhos de DVD, banheiros, automóveis, empregadas domésticas, máquinas de lavar roupa, freezers, aparelho de som e geladeira. Para indicação da quantidade de cada item, o questionário disponibilizava das seguintes alternativas: zero, um, dois, três e quatro ou mais. Também era registrado no questionário o nível educacional do chefe de família, com as opções: analfabeto/até terceira série fundamental, quarta série fundamental, fundamental completo, médio completo e ensino superior completo. Cada resposta gerou um determinado valor em pontos que, no final gera um escore para o participante de acordo com o nível socioeconômico (ABEP, 2010).

No espaço destinado à autopercepção, cada participante deveria se avaliar de acordo com nove características (rosto bonito, corpo bonito, saúde, sociável, bem-humorado, sincero, boa condição financeira, determinado/trabalhador e inteligente), já utilizadas em outros estudos (Castro \& Lopes, 2011; Castro et al., 2012; Castro, Hattori, Yamamoto, \& Lopes, 2014). Para cada característica o participante podia atribuir de 0 a 9 pontos, sendo a quantidade de pontos correspondente a quanto ele julgava ter para cada característica. Havia também uma escala de diferencial semântico no formato de uma reta com 
10 centímetros na qual o participante avaliava, marcando em um ponto da mesma, quão atraente se considerava variando de nada atraente (0) a extremamente atraente (10).

\section{Procedimento}

A pesquisa foi de participação voluntária e visou respeitar o anonimato dos participantes, necessitando que eles se identificassem apenas no Termo de Consentimento Livre e Esclarecido (TCLE), o qual forneceu uma breve introdução a respeito da pesquisa ao participante e quais seriam as atividades desempenhadas caso concordasse em participar. Também foram salientadas questões que fizeram menção à ausência de trocas financeiras envolvidas na pesquisa, à participação voluntária (respondendo aos questionários apenas as pessoas que quisessem fazê-lo, podendo desistir de participar a qualquer momento), e à garantia da integridade e da preservação da identidade dos participantes. O TCLE continha o e-mail do pesquisador responsável para que os participantes interessados em tirar dúvidas ou obter mais informações sobre a pesquisa pudessem entrar em contato para maiores esclarecimentos.

O TCLE foi coletado separadamente do questionário e seguiu as determinações das Diretrizes e Normas Regulamentadoras de Pesquisas Envolvendo Seres Humanos (Conselho Nacional de Saúde [CNS], 2012). Ao concordar em participar da pesquisa, os participantes assinaram o TCLE, ficaram com uma cópia deste e responderam o questionário em seguida.

\section{Análises de dados}

Dez testes modelos lineares gerais (General Linear Models - GLM) 2 (sexo) x 3 (nível econômico) foram realizados (um para cada característica e um para atratividade geral). Dessa forma, foi possível testar se há diferença na autopercepção como parceiro romântico e atratividade geral (variáveis dependentes) de acordo com o nível econômico e/ou o sexo (variáveis independentes) dos participantes. $\mathrm{O}$ critério utilizado para verificar significância foi $p<0,05$. Para as análises post-hoc foi utilizado o teste $t$ para amostras independentes com correção de Bonferroni, para evitar erro do tipo I (Mittelhammer, Judge, \& Miller, 2000).

\section{Resultados}

Os resultados encontrados apontaram diferença significativa da autopercepção da Atratividade geral da interação entre sexo e nível socioeconômico (Tabela 1). Para melhor descrição do resultado encontrado, foram feitos testes $t$ independentes comparando homens e mulheres dentro de cada nível socioeconômico para detalhar a interação da Atratividade geral $\left[t_{(2,54)}=-2,53\right.$; $p=0,015]$, que indicaram que homens de classe A $(M=63,89 ; D P=13,03)$ avaliaram-se como mais atraentes que mulheres de classe $\mathrm{A}$ ( $M=$ $52,89 ; D P=16,51)$. Não foram observadas diferenças significativas para os demais contrastes $(p>0,05)$.

Houve também diferença entre nível econômico para a característica Boa condição financeira (Tabela 1), na qual os participantes com maior nível econômico atribuíram mais pontos para esta característica que participantes de menor nível socioeconômico (Classe A: $M=4,89 ; D P=$ 1,76. Classe B: $M=4,35 ; D P=1,53$. Classe C: $M=3,97 ; D P=1,60)$. Para a característica Determinado e trabalhador, a diferença encontrada foi para sexo, na qual mulheres se consideraram mais determinadas e trabalhadoras $(M=6,94$; $D P=1,86)$ que homens $(M=6,40 ; D P=1,94)$.

\section{Discussão}

À luz da Teoria das Estratégias Sexuais (Buss \& Schmitt, 1993), diferentes pressões evolutivas atuaram sobre homens e mulheres, levando-os a modular suas preferências de parceiro sexual de forma a valorizar mais no sexo oposto as características que favorecem o aumento do sucesso reprodutivo individual. Ao buscar por um parceiro romântico, principalmente para relacionamentos estáveis, a percepção de valor de mercado de cada indivíduo deve ser similar entre o provável casal e, portanto, um dos passos seria a própria avaliação como parceiro romântico 
Tabela 1

Comparação da Autopercepção entre Níveis Socioeconômicos e Sexo

\begin{tabular}{llccccc}
\hline & $g l$ & $F$ & $p$ & $\eta^{2}$ Parcial & Poder \\
\hline Rosto bonito & & & & & & \\
& NSE & 2,310 & 0,06 & 0,944 & $<0,001$ & 0,059 \\
& Sexo & 1,310 & 0,30 & 0,583 & 0,001 & 0,085 \\
& NSE vs Sexo & 2,310 & 0,59 & 0,555 & 0,004 & 0,148
\end{tabular}

Corpo bonito

$\begin{array}{lccccc}\text { NSE } & 2,310 & 1,37 & 0,255 & 0,009 & 0,294 \\ \text { Sexo } & 1,310 & 0,05 & 0,817 & <0,001 & 0,056 \\ \text { NSE vs Sexo } & 2,310 & 1,52 & 0,221 & 0,010 & 0,322\end{array}$

Saúde

$\begin{array}{llllll}\text { NSE } & 2,310 & 0,58 & 0,563 & 0,004 & 0,145 \\ \text { Sexo } & 1,310 & 1,47 & 0,227 & 0,005 & 0,227 \\ \text { NSE vs Sexo } & 2,310 & 1,44 & 0,238 & 0,009 & 0,308\end{array}$

Sociável

$\begin{array}{lccccc}\text { NSE } & 2,310 & 0,58 & 0,563 & 0,004 & 0,145 \\ \text { Sexo } & 1,310 & 0,05 & 0,827 & <0,001 & 0,055 \\ \text { NSE vs Sexo } & 2,310 & 0,50 & 0,606 & 0,003 & 0,132\end{array}$

Bem humorado

$\begin{array}{llllll}\text { NSE } & 2,310 & 0,81 & 0,444 & 0,005 & 0,189 \\ \text { Sexo } & 1,310 & 0,36 & 0,550 & 0,001 & 0,092 \\ \text { NSE vs Sexo } & 2,310 & 0,10 & 0,904 & 0,001 & 0,065\end{array}$

Sincero

$\begin{array}{lccccc}\text { NSE } & 2,310 & 0,11 & 0,892 & 0,001 & 0,067 \\ \text { Sexo } & 1,310 & 0,02 & 0,878 & <0,001 & 0,053 \\ \text { NSE vs Sexo } & 2,310 & 1,71 & 0,183 & 0,011 & 0,357\end{array}$

Boa condição financeira

$\begin{array}{lccccc}\text { NSE } & 2,310 & 5,13 & \mathbf{0 , 0 0 6} & 0,032 & 0,822 \\ \text { Sexo } & 1,310 & 0,05 & 0,826 & <0,001 & 0,056 \\ \text { NSE vs Sexo } & 2,310 & 0,22 & 0,806 & 0,001 & 0,084\end{array}$

Determinado/trabalhador

$\begin{array}{lrrrrr}\text { NSE } & 2,310 & 2,42 & 0,091 & 0,015 & 0,486 \\ \text { Sexo } & 1,310 & 4,93 & \mathbf{0 , 0 2 7} & 0,016 & 0,600 \\ \text { NSE vs Sexo } & 2,310 & 0,04 & 0,962 & <0,001 & 0,056\end{array}$

Inteligente

$\begin{array}{llllll}\text { NSE } & 2,310 & 0,46 & 0,634 & 0,003 & 0,124 \\ \text { Sexo } & 1,310 & 0,45 & 0,502 & 0,001 & 0,103 \\ \text { NSE vs Sexo } & 2,310 & 1,47 & 0,232 & 0,009 & 0,313\end{array}$

Atratividade geral

\begin{tabular}{llllll} 
NSE & 2,310 & 0,31 & 0,736 & 0,002 & 0,099 \\
Sexo & 1,310 & 0,68 & 0,412 & 0,002 & 0,130 \\
NSE vs Sexo & 2,310 & 3,58 & $\mathbf{0 , 0 2 9}$ & 0,023 & 0,661 \\
\hline
\end{tabular}

Nota. Gl: grau de liberdade; NSE: Nível socioeconômico; Valores significativos destacados em negrito. 


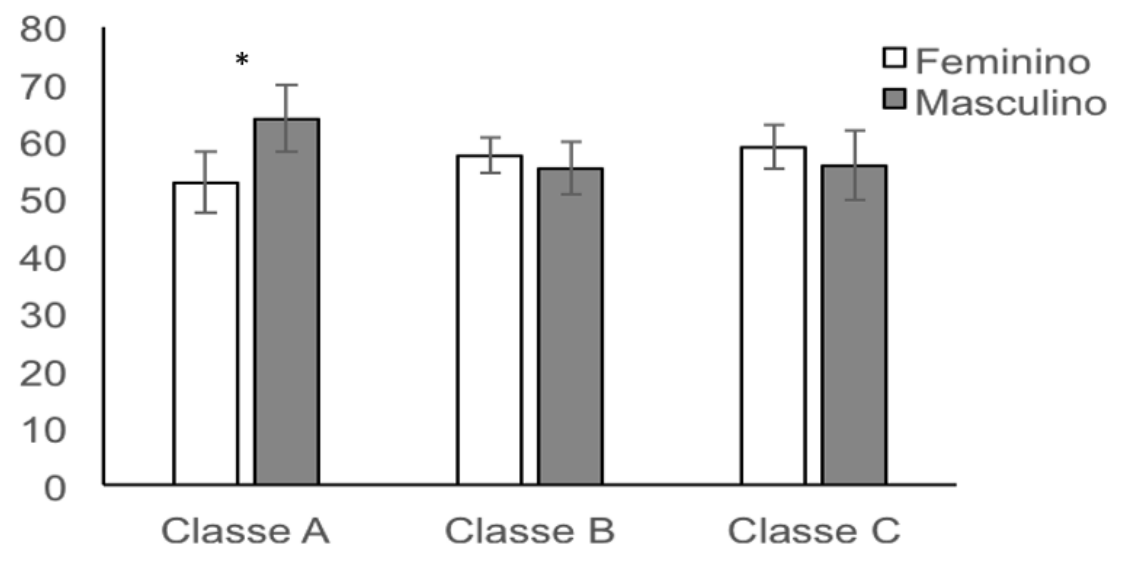

Figura 1. Médias e Desvios padrão Femininos e Masculinos da Autopercepção de Atratividade Geral nos Diferentes Níveis Socioeconômicos.

Nota. *médias significativamente diferentes $(p<0,05)$.

(Buston \& Emlen, 2003; Castro et al., 2012; Lee et al., 2008).

No geral, as características mais valorizadas pelo sexo oposto são aquelas mais importantes para a autopercepção como parceiro romântico (Buston \& Emlen, 2003; Castro et al., 2012; Mafra \& Lopes, 2014), sendo esta um fator que influencia a escolha de parceiro romântico. Porém, ela está sujeita a variações, principalmente quando se leva em consideração o sexo e o ambiente no qual o indivíduo está inserido, devido à variação da disponibilidade e do valor de mercado dos competidores (Gangestad \& Simpson, 2000). Desse modo, em um contexto com pessoas de diferentes níveis socioeconômicos, a autopercepção masculina tenderia a se modificar em função dessa variável, uma vez que status social é uma das características mais valorizadas pelas mulheres ao procurar por um parceiro romântico (Buss \& Shackelford, 2008; Fisher et al., 2008; Lee et al., 2008; Lippa, 2007).

$\mathrm{Na}$ presente pesquisa, embora cada participante tivesse um determinado nível socioeconômico, todos eles encontravam-se dentro de um mesmo contexto: a universidade. Nossos resultados, corroboram com a perspectiva supracitada ao evidenciar que homens que estão inseridos em um nível socioeconômico mais elevado (classe A) consideram-se mais atraentes em geral como parceiros românticos quando comparados às mulheres. Além disso, os resultados dão suporte à Teoria do Pluralismo Estratégico (Gangestad \&
Simpson, 2000) uma vez que homens de maior nível socioeconômico se consideram também mais atraentes que homens de menor nível socioeconômico (efeito ambiental).

Houve, também, diferença entre nível econômico para a característica Boa condição financeira, indicando que provavelmente devido à perspectiva de futuro ser semelhante para toda a amostra, quando inseridos em um ambiente universitário, essa discrepância é atenuada e a diferença de autopercepção torna-se perceptível apenas para a característica relativa a status social que mensura diretamente a quantidade de recursos disponíveis no momento atual do participante e não quanto às outras características relativas a status social (inteligência e determinado/trabalhador) que dão uma perspectiva de ascensão social futura.

Mesmo que a autopercepção tenha sido similar para homens e mulheres para a maioria das características, o que vai de encontro com a maioria das pesquisas realizadas com parceiros românticos (ex. Buss \& Shackelford, 2008; Fisher et al., 2008; Goodwin et al., 2012; Gutierres et al., 1999), as mulheres perceberam-se como mais determinadas/trabalhadoras que os homens. No Brasil, houve uma diminuição na desigualdade de gênero nos anos 90, no entanto, as taxas de desemprego são maiores entre mulheres, os acessos a cargos de liderança são mais limitados e as mulheres ainda ganham menores salários que homens ao desempenhar as mesmas 
funções, além das elevadas cargas de trabalho e da baixa proteção social feminina (Melo, 2005; Santana, 2010). Nesta perspectiva, este resultado ressalta a necessidade de elas trabalharem mais que eles para poderem ter seu espaço no ambiente de trabalho.

O presente estudo revelou que as mulheres ressaltam a necessidade de serem determinadas e trabalhadoras, enquanto que em outros estudos os homens que afirmam tais características como sendo mais importantes para a autopercepção como parceiro romântico. Tal resultado pode ser proveniente da diferença da igualdade de gênero existente entre os países investigados e o Brasil. Dados do Human Development Report (2015), corroboram tal justificativa ao revelar o elevado índice de desigualdade de gênero no Brasil (GII $=0,414$ ) comparado com outros países investigados (por exemplo, Canadá; Fisher et al., 2008) com GII $=0,098$, Noruega (Grøntvedt \& Kennair, 2013), com GII $=0,053$.

Adicionalmente, nossos resultados complementam achados de Mafra e Lopes (2014), os quais indicaram que pessoas de diferentes níveis socioeconômicos, inferidos pelo nível educacional, possuem autopercepção como parceiro romântico diferente. Porém, no referido estudo não houve a comparação entre as classes socioeconômicas dentro de um mesmo nível educacional, o que possibilitaria a comparação de autopercepção que estão inseridas em um mesmo contexto social, mas que possuem níveis socioeconômicos distintos.

Embora as nove características abordadas no trabalho sejam representantes de três das dimensões mais valorizadas na busca por parceiros românticos (rosto bonito, corpo bonito e saúde como representantes de atratividade física; boa condição financeira, inteligente e determinado/ trabalhador como representantes de status social; e sociável, bom humor e sinceridade como representante de habilidade social; Castro \& Lopes, 2011; Castro et al., 2012; Castro et al., 2013; Castro et al., 2014; Mafra, Castro, \& Lopes, 2015; Mafra \& Lopes, 2014) e a atratividade geral forneça informação à respeito de todas as características avaliadas conjuntamente, outras características como ativo, assertivo, atencioso ao parceiro, desejo por crianças ou independente podem também ser levadas em consideração (Kirsner, Figueredo, \& Jacobs, 2003) e possuir pesos distintos quando a avaliação de um parceiro romântico é realizada, sendo, desta forma, importantes também para a avaliação desta autopercepção.

Outra limitação do estudo é a ausência de classes mais baixas (classes D e E) para a comparação de todos os níveis socioeconômicos existentes no Brasil. Este problema seria solucionado ao ampliar e diversificar mais a amostra. No entanto, como procuramos trabalhar com participantes dentro de um mesmo contexto, essas classes foram sub-representadas, não possibilitando maiores explorações a níveis confiáveis da amostra. A mesma limitação ocorreu quanto aos participantes não-heterossexuais. Como as preferências de parceiros românticos mudam de acordo com a orientação sexual (ver Lippa, 2007 para uma revisão), os participantes não-heterossexuais foram retirados da amostra.

Além disso, a pesquisa aqui apresentada foi desenvolvida apenas em uma cidade do Brasil, o que não possibilita traçar um panorama de tais diferenças de autopercepção em relação aos níveis socioeconômicos a nível nacional. Para isso, sugere-se que um estudo mais abrangente seja feito, coletando dados em todas as regiões do país. Sugere-se também que estudos futuros investiguem se há diferença de autopercepção como parceiro romântico entre participantes não-heterossexuais de diferentes níveis socioeconômicos.

\section{Conclusão}

Ainda que os resultados tenham apontado que homens e mulheres são sensíveis a percepção de condição financeira do ambiente, tal efeito foi mais pronunciado entre os homens, confirmando que o nível socioeconômico é um fator que influencia na autopercepção masculina, provavelmente devido ao fato de que mulheres tendem a adotar mais estratégias de longo prazo e valorizar mais características em um parceiro em potencial que indicam o investimento de recursos na prole. Por outro lado, a nossa amos- 
tra comportou-se diferentemente da maioria dos resultados encontrados para outras populações ao mostrar que mulheres se consideram mais determinadas/trabalhadoras que homens, o que pode ser reflexo do alto índice de desigualdade de gênero existente no país de estudo, fazendo com que mulheres precisem trabalhar mais que homens para garantir seu espaço no mercado de trabalho.

\section{Referências}

Associação Brasileira de Empresas de Pesquisa. (2010). Dados com base no levantamento socioeconômico de 2010 - IBOPE. Recuperado em www.abep.org/Servicos/Download.aspx?id=05

Buss, D. M. (1989). Sex differences in human mate preferences: Evolutionary hypotheses tested in 37 cultures. Behavioral and Brain Science, 12, $1-49$.

Buss, D. M., \& Schmitt, D. P. (1993). Sexual strategies theory: An evolutionary perspective on human mating. Psychological Review, 100, 204-232. doi: 10.1037/0033-295X.100.2.204

Buss, D. M., \& Shackelford, T. K. (2008). Attractive women want it all: Good genes, economic investment, parenting proclivities, and emotional commitment. Evolutionary Psychology, 6(1), 134-146.

Buston, P., \& Emlen, S. (2003). Cognitive processes underlying human mate choice: The relationship between self-perception and mate preference in Western society. Proceedings of the National Academy of Sciences, 100(15), 8805-8810. doi: $10.1073 /$ pnas. 1533220100

Castro, F. N., \& Lopes, F. A. (2011). Romantic preference in Brazilian undergraduate students: From the short term to the long term. Journal of Sex Research, 47, 1-7. doi: 10.1080/00224499.2010.506680

Castro, F. N., Hattori, W. T., \& Lopes, F. A. (2012). Relationship maintenance or preference satisfaction? Male and female strategies in romantic partner choice. Journal of Social, Evolutionary, and Cultural Psychology, 6(2), 217-226.

Castro, F. N., Hattori, W. T., Yamamoto, M. E., \& Lopes, F.A.(2013). Romantic partners in a market perspective: Expectations about what ensures a highly desirable partner. Psychological Reports:
Relationships \& Communications, 113(2), 605618. doi: $10.2466 / 21.19 . P R 0.113 \times 23 z 6$

Castro, F. N., Hattori, W. T., Yamamoto, M. E., \& Lopes, F. A. (2014). Social comparisons on self-perception and mate preferences: The self and the others. Psychology, 5, 688-699. doi: 10.4236/psych.2014.57080

Central Intelligence Agency. (2014). The World Factbook. Retrieved from https://www.cia.gov/ library/publications/the-world-factbook/geos/ br.html

Conselho Nacional de Saúde. (2012). Resolução $n^{o}$ 466, de 12 de dezembro de 2012. Dispõe sobre diretrizes e normas regulamentadoras de pesquisas envolvendo seres humanos. Recuperado em http://conselho.saude.gov.br/resolucoes/2012/ Reso466.pdf

Dosmukhambetova, D., \& Manstead, A. (2011). Strategic reactions to unfaithfulness: Female self-presentation in the context of mate attraction is linked to uncertainty of paternity. Evolution and Human Behavior, 32, 106-117. doi: 10.1016/j.evolhumbehav.2010.08.006

Fisher, M., Cox, A., Bennett, S., \& Gavric, D. (2008). Components of self-perceived mate value [Special Issue: Proceedings of the $2^{\text {nd }}$ Annual Meeting of the North-Eastern Evolutionary Psychology Society]. Journal of Social, Evolutionary, and Cultural Psychology, 2(4), 156-168.

Gangestad, S. W., \& Simpson, J. A. (2000). The evolution of human mating: Trade-offs and strategic pluralism. Behavioral and Brain Sciences, 23, 573-644. doi: 10.1017/S0140525X0000337X

Gaulin, S. J., \& McBurney, H. D. (2001). Psychology: An evolution approach. Englewood Cliffs, NJ: Prentice Hall.

Geary, D., Vigil, J., \& Byrd-Craven, J. (2004). Evolution of human mate choice. Journal of Sex Research, 41, 27-42. doi: 10.1080/00224490409552211

Goodwin, R., Marshall, T., Fülöp, M., Adonu, J., \& Spiewak, S. (2012). Mate value and self-esteem: Evidence from eight cultural groups. PLoS ONE, 7(4), e36106. doi: 10.1371/journal. pone. 0036106

Grøntvedt, T. V., \& Kennair, L. E. O. (2013). Age preferences in a gender egalitarian society. Journal of Social, Evolutionary, and Cultural Psychology, 7(3), 239-249. 
Gutierres, S., Kenrick, D., \& Partch, J. (1999). Beauty, dominance, and the mating game: Contrast effects in self-assessment reflect gender differences in mate selection. Personality and Social Psychology Bulletin, 25(9), 1126-1134. doi: $10.1177 / 01461672992512006$

Hattori, W. T., Castro, F. N., \& Lopes, F. A. (2013). Mate choice in adolescence: Idealizing romantic partners. Psico, 44, 226-234.

Heo, S. Moser, D. K., Chung, M. L., \& Lennie, T. A. (2012). Social status, health-related quality of life, and event-free survival in patients with heart failure. European Journal of Cardiovascular Nursing, 11(2), 141-149. doi: 10.1016/j. ejcnurse.2010.10.003

Human Development Report. (2015). Gender Inequality Index (GII). Retrieved from http://hdr. undp.org/en/content/gender-inequality-index-gii

Instituto Brasileiro de Geografia e Estatística. (2010). Síntese de indicadores sociais: Uma análise das condições de vida da população brasileira. Estudos \& Pesquisas: Informação Demográfica e Socioeconômica, 27.

Kenrick, D. T., Sadalla, E. K., Groth, G., \& Trost, M. R. (1990). Evolution, traits, and the stages of human courtship: Qualifying the parental investment model. Journal of Personality, 58(1), 97116. doi: 10.1111/j.1467-6494.1990.tb00909.x

Kirsner, B. R., Figueredo, A. J., \& Jacobs, W. J. (2003). Self, friends, and lovers: Structural relations among Beck Depression Inventory scores and perceived mate values. Journal of Affective Disorders, 75, 131-148. doi: 10.1016/S01650327(02)00048-4

Kruger, D., Fitzgerald, C., \& Peterson, T. (2010). Female scarcity reduces women's marital ages and increases variance in men's marital ages. Evolutionary Psychology, 8(3), 420-431. doi: https:// doi.org/10.1177/147470491000800309

Lee, L., Loewenstein, G., Ariely, D., Hong, J., \& Young, J. (2008). If I'm not hot, are you hot or not? - Physical attractiveness evaluations and dating preferences as a function of one's own attractiveness. Psychological Science, 19(7), 669677. doi: 10.1111/j.1467-9280.2008.02141.x
Lippa, R. A. (2007). The preferred traits of mates in a cross-national study of heterosexual and homosexual men and women: An examination of biological and cultural influences. Archives of Sexual Behavior, 36, 193-208. doi:10.1007/ s10508-006-9151-2

Mafra, A. L., Castro, F. N., \& Lopes, F. A. (2015). Investment in beauty, exercise, and self-esteem: Are they related to self-perception as a romantic partner? Evolutionary Psychological Science, 2(1), 24-31. doi: 10.1007/s40806-015-0032-6

Mafra, A. L., \& Lopes, F. A. (2014). “Am I good enough for you?" Features related to selfperception and self-esteem of Brazilians from different socioeconomic status. Psychology, 5(7), 653-663. doi: 10.4236/psych.2014.57077

Melo, H. P. (2005). Gênero e pobreza no Brasil (Relatório Final do Projeto Governabilidad Democratica de Género en America Latina y el Caribe). Brasília, DF: Comissão Econômica para a América Latina e o Caribe.

Mittelhammer, R. C., Judge, G. G., \& Miller, D. J. (2000). Econometric Foundations. Cambridge, MA: Cambridge University Press.

Pawlowski, B. (2000). The biological meaning of preferences on the human mate market. Anthropological Review, 63, 39-72.

Santana, A. M. (2010). Mulher mantenedora/homem chefe de família: Uma questão de gênero e poder. Forum Identities Magazine, 4, 71-87.

The Hofstede Centre. (2013). National Culture. Retrieved from http://geert-hofstede.com/brazil. html

Trivers, R. (1972). Parental investment and sexual selection. In B. Campbell (Ed.), Sexual selection and the descent of man (pp. 136-179). Chicago, IL: Aldine-Atherton. restrições em qualquer meio, desde que você dê crédito apropriado ao(s) autor(es) original(ais) e à fonte, fornecer um link para a licença Creative Commons e indicar se as alterações foram feitas. 